\title{
High sensitivity wake-up radio using spreading codes: design, evaluation, and applications
}

\author{
Wen-Chan Shih" ${ }^{*}$, Raja Jurdak², Bih-Hwang Lee ${ }^{1}$ and David Abbott ${ }^{3}$
}

\begin{abstract}
Most of the published wake-up radios propose low energy design at the expense of reduced radio range, which means that they require an increased deployment density of sensor networks. In this article, we introduce a design of a high sensitivity $916.5 \mathrm{MHz}$ wake-up radio using low data rate and forward error correction (FEC). It improves the sensitivity, up to $-122 \mathrm{dBm}$ at a data rate $370 \mathrm{bit} / \mathrm{s}$. It achieves up to $13 \mathrm{~dB}$ of coding gain with symbol error rate (SER) $10^{-2}$, and up to 4 times the range of the data radio, rendering it more suitable to sensor networks. Our design can receive wake-up signal reliably from any IEEE 802.15.4 transmitter and achieves a low packet error rate (PER) 0.0159 at SNR 4 dB. Furthermore, our design encodes the node ID into a wake-up signal to avoid waking up the undesired nodes.
\end{abstract}

Keywords: Wake-up radio, Wireless sensor network applications, Forward error correction (FEC)

\section{Introduction}

The sensor node has constrained energy resources, and the radio accounts for a major portion of the node's energy budget $[1,2]$. Current research, into energy efficiency in sensor networks, puts the radio in sleep mode when there is no traffic to reduce energy consumption. These works can be classified into two main categories: (1) MAC protocols [2-6]; and (2) wake-up radios [6-14].

Current MAC protocols do not eliminate idle listening. Event-driven wake-up radios provide an opportunity to solve idle listening. Previously published wake-up radios are low power with low sensitivity [6-14]. They provide extremely low energy consumption at the cost of shorter read range than the data radio [15]. They effectively limit the data radio range. As low power wake-up radio provides short radio ranges, senders must be within a short distance away to trigger the wake-up radio. Because the wake-up range is typically much smaller than the data radio's communication range, the use of wake-up radios constrains the data communication range. This in turn effectively increases the deployment density, which is not suitable to sensor networks.

\footnotetext{
* Correspondence: teddyshihau@gmail.com

'Department of Electrical Engineering, National Taiwan University of Science and Technology, 2F, EE, No. 43, Sec. 4, Keelung Rd, Taipei 106, Taiwan Full list of author information is available at the end of the article
}

Our article addresses this issue by proposing a design of a high sensitivity wake-up radio circuit with forward error correction (FEC), which achieves a longer radio range and is more reliable than the IEEE 802.15.4 compliant data radio. It also reduces the deployment density and is more suitable to sensor networks than other low sensitivity wake-up radios. Our design has a tradeoff with energy and latency $[11,14,16,17]$.

Our design enables a new class of applications that can benefit from low rate telemetry at enhanced radio ranges, such as military applications, hospital applications, emergency services, and hidden node explorations' system services. To evaluate the performance of our design, we characterize sensitivity and symbol error rate (SER) in theoretical analysis, simulations and empirical experiments.

The novel contributions of this article are threefold:

- Proposal of the high sensitivity wake-up radio design that improves sensitivity, reduces the deployment density, is more reliable and suitable to sensor networks than other low sensitivity wake-up radios. Our design enables the potential applications for sensor networks.

- Presentation of the high sensitivity wake-up radio circuit design and implementation that utilizes OnOff Keying (OOK) demodulation, low data rate and

\section{SpringerOpen ${ }^{\odot}$}

(C) 2011 Shih et al; licensee Springer. This is an Open Access article distributed under the terms of the Creative Commons Attribution License (http://creativecommons.org/licenses/by/2.0), which permits unrestricted use, distribution, and reproduction in any medium, provided the original work is properly cited. 
FEC to achieve target high sensitivity of $-122 \mathrm{dBm}$ and long communication range of $1 \mathrm{~km}$.

- Performance evaluation of OOK modulation and FEC through theoretical analysis, simulations and empirical experiments. Provision of guidelines based on evaluation results for FEC scheme's configuration according to the SER and the radio range of the target application.

The remainder of the paper is organized as follows. Section 2 presents our high sensitivity wake-up radio design and its applications. Section 3 evaluates the performances of our design. Section 4 discusses the results and concludes the article.

\section{Related work}

The wake-up radios that have been proposed for wireless sensor networks can be classified into two main categories:

- Passive wake-up radio. Passive wake-up radios use passive diodes to build the envelope detector that rectifies the RF signal to the baseband signal to detect data. Some of them use the charge pump circuit to accumulate the energy from the RF signal to generate a trigger signal that interrupts the receiver's microprocessor [18-21].

- Active wake-up radio. The active wake-up radios use filters, amplifiers, and specific modulation methodologies, such as PPM or PWM, to amplify the desired RF signal and suppress the noise to improve the sensitivity. The amplifier accounts for a major portion of the power dissipation in the active wakeup radio. As modulation methodologies deliver different BERs at a given SNR, they determine the system performance and sensitivity. While our design is also active, it uses FEC scheme, which previous work does not consider [10-15], in order to improve the sensitivity. In terms of the data radio for wake-up radios [12,14,15], the [12] requires a specific $2 \mathrm{GHz}$ transmitter to be its data radio. However, our design and $[14,15]$ can use an off-the-shelf IEEE 802.15.4 radio to be the data radio. Although our design and $[14,15]$ use the IEEE 802.15.4 radio as the wake-up signal sender, we can use it as the wake-up signal listener and compare our design with it to demonstrate our design has higher sensitivity than it.

The work in [22] proposes a special node, which includes a sensor node coupled with a radio-frequency identification (RFID) reader. As the RFID reader of the special node has a fixed short read range, the special node might not detect some active and passive tags. Finally, their use of a special node limits the network scalability. In contrast, our wake-up radio provides various sensitivity configurations to achieve multiple read ranges in one sensor node without RFID reader. Our wake-up design with up to 4 times the range of data radio enables the mitigation of the hidden node problem to reveal hidden nodes.

The work in [11] proposes a simple wakeup radio using the standard ZigBee chip with OOK modulation. It has a low sensitivity of $-30 \mathrm{dBm}$ for achieving the power consumption of $33 \mu \mathrm{W}$ and less than $0.6 \mathrm{~m}$ read range. However, our work similarly uses the continuous transmission mode of the IEEE 802.15.4 compliant radio chip with OOK modulation. Our design achieves higher sensitivity $-122 \mathrm{dBm}$ using FEC scheme for a reliable and longer communication range. We can also employ a duty cycle (DC) approach [3] to reduce power consumption of our wake-up radio.

The work in [23] proposes a mobile agent middleware and evaluates a fire tracking application. The mobile agent comprises MICA2 motes, TinyOS, and Agilla Middleware. The MICA2's RF transceiver provides a sensitivity of $-97 \mathrm{dBm}$ at $38.4 \mathrm{kBaud}$ with BER $10^{-3}$. The mobile agents are particularly susceptible to message loss that introduces delay. However, our high sensitivity wake-up radio provides higher sensitivity $-122 \mathrm{dBm}$ at SER $10^{-2}$ that reduce hops and latency for fire tracking application. We also use the FEC scheme to suppress the packet error rate (PER) and provide the reliability to reduce the latency.

The work in [24] uses the side channel leakage communication technique to detect relay attack with timingbased protocol for ISO 14443 smart cards. The symmetric key based timing-based protocol is computationally efficient enough to be implemented in resourceconstrained devices. The phenomena of side channel leakage provide the low latency to detect relay attacks within inexpensive implement. However, our design uses the FEC communication technique and low data rate to achieve the long-range communication for the sensor nodes to snoop and collect the information over the air.

The work in [25] proposes a technology to reduce the idle power of a PDA-based phone to increase the battery life time. The prototype includes the MiniBricks and the SmartBricks. The MiniBrick, as a wake-up radio, is connected to the PDA-based phone. It waits for the POWER_ON command from the SmartBrick when the PDA-based phone turns off. The MiniBrick using TR1000 has a short transmitting distance about 30 feet and a long latency about 5 to $10 \mathrm{~s}$. Thus, it needs a large number of infrastructure SmartBricks. However, our design has a longer communication range and a shorter latency than the MiniBrick. Our design also reduces the deployment density. 


\section{High sensitivity wake-up radio Overview}

This article provides the high sensitivity wake-up radio. It can have a longer wake-up range, up to 4 times the data radio's radio range. It also can use IEEE 802.15.4 radio to generate wake-up signals. Before discussing the details of our design, we will look at the motivation of our high sensitivity wake-up radio.

\section{Motivating applications}

Our high sensitivity design provides a long wake-up range. This longer range enables new potential applications which can be categorized under some general headlines: transmit power control, hidden node detection, and security.

- Transmit power control. Nodes can decrease their transmission power to avoid interfering with neighbors, by relying on the high sensitivity wakeup radio to detect these neighbors. In Figure 1, N1 detects activities, it will refrain from using its power amplifier to reduce interference and save energy. This scenario is applicable for hospitals where critical equipment can be highly sensitive to interference. Using our high sensitivity wake-up radio for long range detection, this can be achieved.

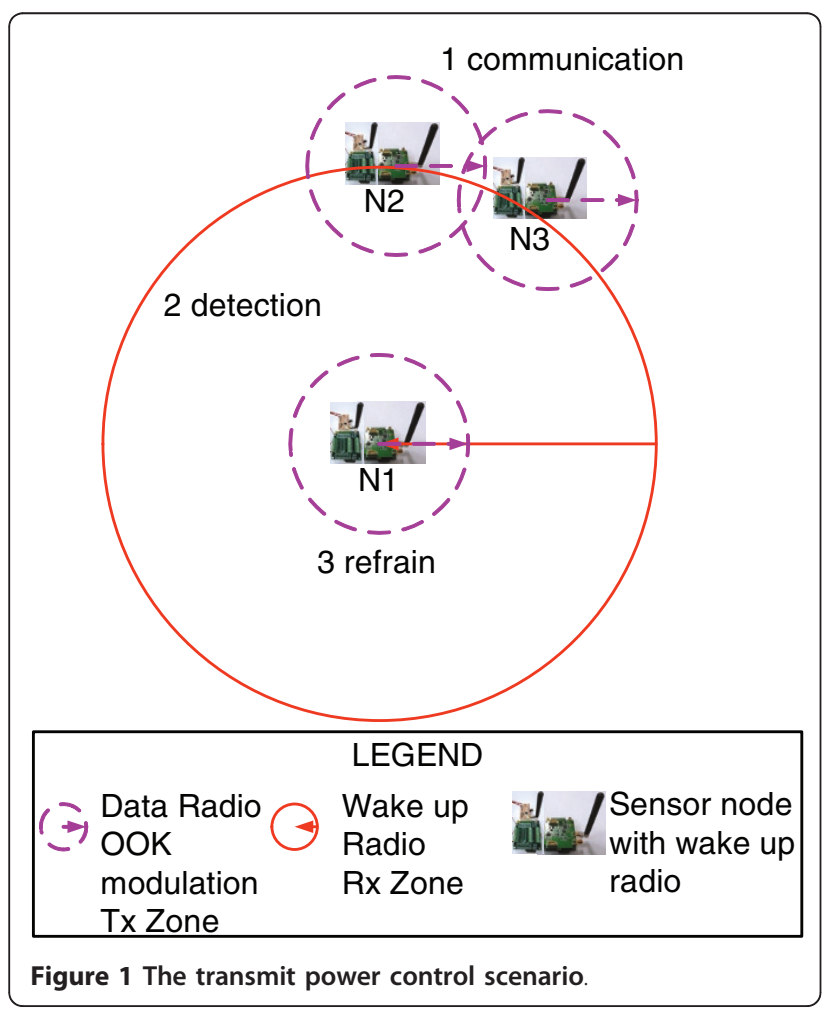

- Hidden node detection. In Figure 2, the receiver $\mathrm{R}$ detects nodes $\mathrm{N} 1$ and $\mathrm{N} 2$ in its vicinity, but $\mathrm{N} 1$ and $\mathrm{N} 2$ are out of each other's communication range. If both nodes (N1 and N2) send data to R simultaneously, a collision occurs. The first of the two nodes (N1 and R) to communicate can use the longrange wake-up radio as an out-of-band reservation channel. This will ensure that any other node (N2) that can hear the wakeup signal will refrain from transmitting concurrently.

- Security. The privacy scenario is a problem for sensor network applications [24]. Our design attacks the privacies of sensor nodes. In Figure 3, when N1 senses other nodes' activities, it turns off the transmission power to snoop on the metadata exchanges without being detected.

\section{Circuit design}

The overview of our wake-up radio design is depicted in Figure 4. The sender node consists of a micro-controller unit (MCU) and IEEE 802.15.4 data radio. The MCU encodes the data with a spreading code and uses the data radio to send an OOK modulation data sequence to the receiver node. As with previous wake up radio proposals, we select OOK modulation rather than more complex schemes, since it requires simple hardware and low implementation cost. The spreading code scheme consists of 16 chips for each pattern (symbol A or symbol B). Symbols A and B represent binary 1 and 0 , respectively. The receiver node employs the wake-up

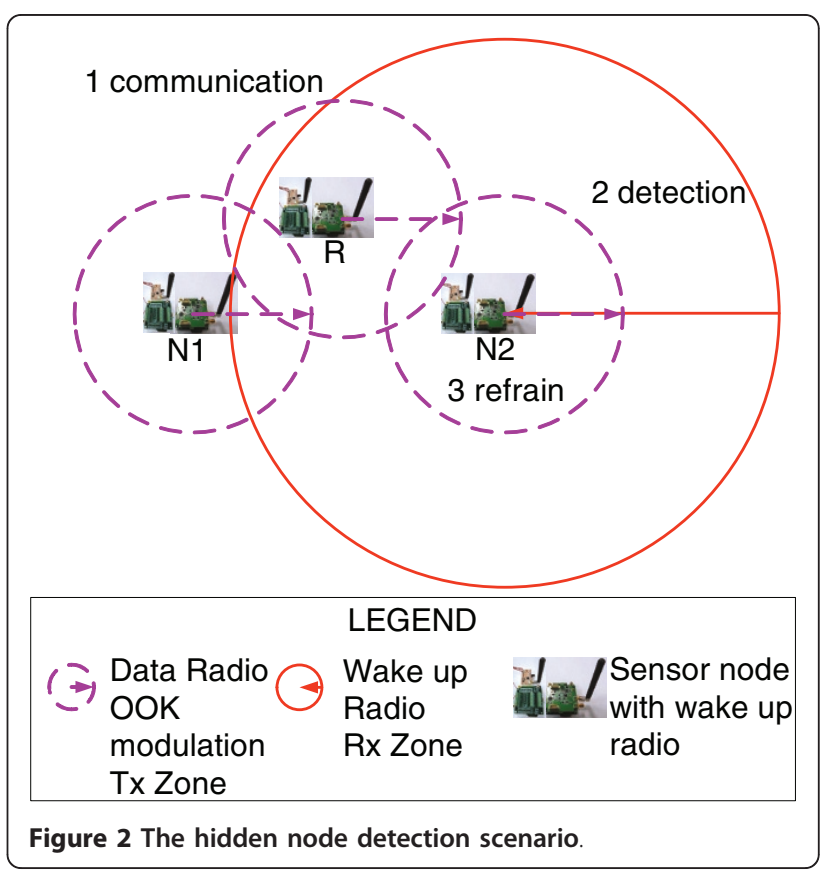




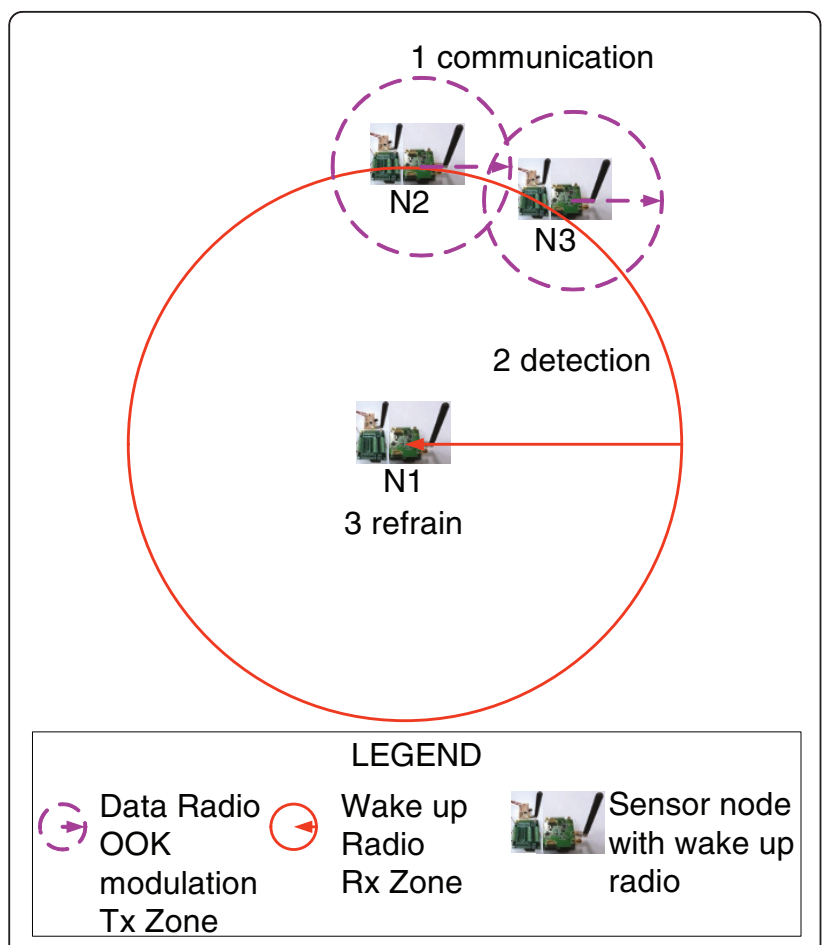

Figure 3 The privacy scenario.

radio to decode the signal using the same spreading code to obtain the wake-up bit sequence.

Our wake-up radio prototype is separate from the sensor node with a built-in main data receiver. It includes a Fleck3b [26] circuit board and an off-the-shelf OOK receiver QwikRadio [27] circuit board. The OOK receiver includes the image reject filter, amplifier, AGC, and OOK demodulation. The OOK receiver circuit board includes the impedance matching, band pass filter for
OOK receiver. The demodulation bandwidth's configuration can be adjusted through jumpers and capacitors in the OOK receiver circuit board. The demodulation bandwidth is set at $6.85 \mathrm{kHz}$ with $22 \mathrm{nF}$ and $1 \mu \mathrm{F}$ capacitors. The crystal, the reference clock at $14.29983 \mathrm{MHz}$ for all the OOK receiver's internal circuits, provides the carrier frequency at $916.5 \mathrm{MHz}$. In order to improve the sensitivity, our wake-up radio prototype uses a Fleck3b circuit board to process spreading code algorithm.

\section{Spreading code algorithm}

We use the spreading code with soft decoding to enable high sensitivity feature. The spreading code detection algorithm for the system model is shown in Additional file 1, Algorithm 1. The sender generates data and sends it, using the proposed spreading code and OOK modulation, to the wake-up radio. We assume the Additive White Gaussian Noise (AWGN) channel which is added into transmitted signals. The wake-up radio receives the signals and uses OOK demodulation to get the binary data sequence. The AWGN noise is suppressed by 4 times oversampling. We choose the factor of 4 empirically as a proof of concept. The characterization of the optimal oversampling factor is described in the second paragraph of the Sect. 4.2.2. The correlation values $C$ are calculated by the oversampling values and the ideal spreading code pattern. As the correlation values $C$ include the AWGN noise portion, the wake-up radio uses a low pass filter (LPF) to suppress the noise to reduce the undesired peak values. The wake-up radio uses the finding local maximum correlation algorithm to determine the valid correlation values. Based on the valid correlation values, the wake-up radio determines the detected symbols and timing recovery. For empirical performance evaluation purposes, we calculate the SER
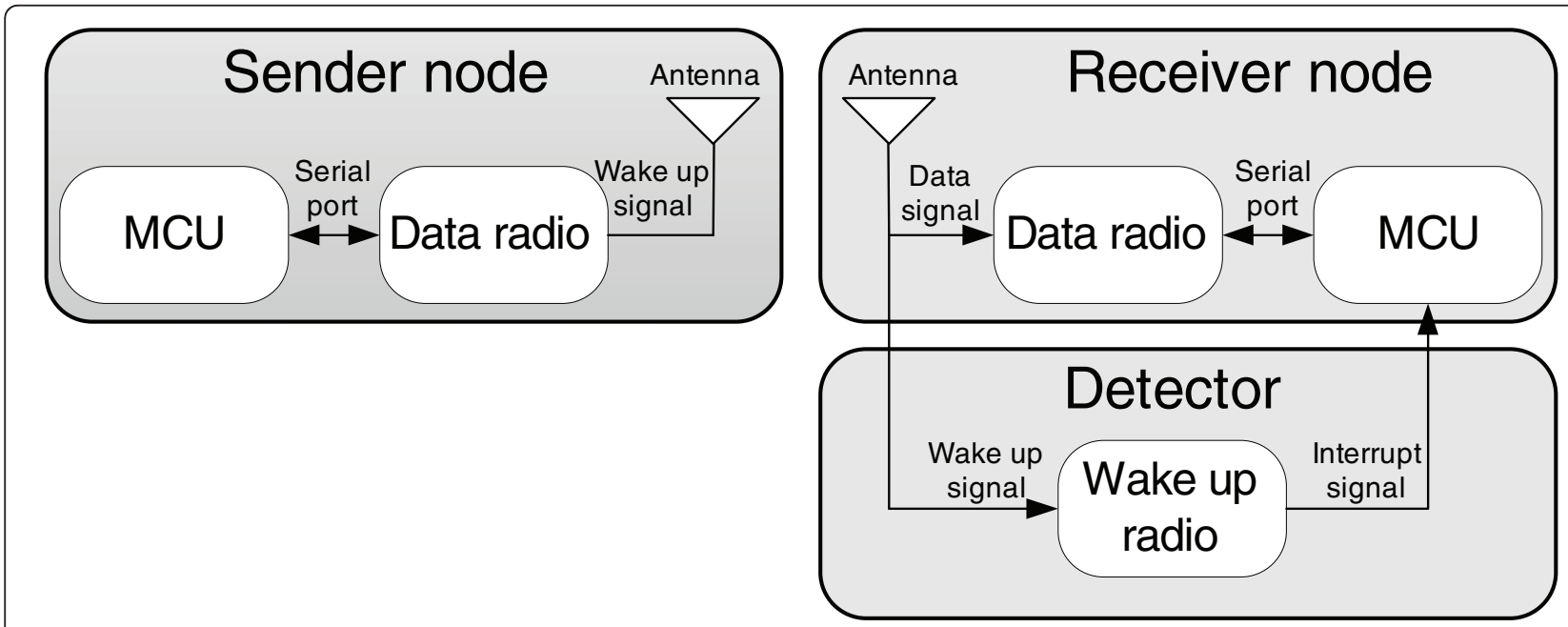

Figure 4 Overview of our wake-up radio 
values for different symbols by the comparison of the data source with the detected data.

\section{Theoretical analysis}

In this section, we analyze the performance characteristics of the proposed spreading code scheme. The spreading code scheme uses system model to analyze the symbol error probability of the spreading code scheme.

\section{Upper bound of symbol error probability analysis}

Our spreading code can be modeled by the constantweight Hadamard code model [28]. Our spreading code is defined as

$c_{i} \in\{0,1\}, i \in\{1,2,3, \ldots, 16\},\left\{c_{i}\right\}=\{1111111100000000\}$ for symbol A $d_{i} \in\{0,1\}, i \in\{1,2,3, \ldots, 16\},\left\{d_{i}\right\}=\{0000000011111111\}$ for symbol B

It uses $N_{\text {cps }}$ chips per symbol and $N_{\text {os }}$ times oversampling to suppress the noise. The $N_{\text {cps }}$ is the number of the cells per code word and $N_{\mathrm{os}}$ reduces the noise variance (noise power) by a factor $1 / N_{\text {os }}$. Our spreading code can be represented as the 2-ary Hadamard code $H$ $\left(N_{\text {cps }}, 1\right)$ waveforms with diversity $\frac{1}{2} d_{\min }=\frac{1}{4} \cdot n=\frac{N_{\text {cps }}}{4}$, $M=2, k=1$, and $n=N_{\text {cps }}$ cells. The SNR per cell is given by

$$
\mathrm{SNR}_{\mathrm{c}}=\frac{2}{N_{\text {cps }}} \cdot \mathrm{SNR}_{\mathrm{b}} \cdot \frac{1}{1 / N_{\mathrm{os}}} \cdot \frac{N_{\text {cps }}}{N_{\text {cps_base }}}=2 \cdot \mathrm{SNR}_{\mathrm{b}} \cdot N_{\mathrm{os}} \cdot \frac{1}{N_{\text {cps_base }}}
$$

where the SNR per bit $\mathrm{SNR}_{\mathrm{b}}$ is related to the SNR per cell $\mathrm{SNR}_{\mathrm{c}}$ and the $\frac{N_{\mathrm{cps}}}{N_{\text {cps_base }}}$ is the scale value to keep the constant $\mathrm{SNR}_{\mathrm{c}}$ when $N_{\mathrm{cps}}$ increases. The probability of error for two orthogonal waveforms with diversity $P_{2}\left(\frac{1}{2} d_{\min }\right), P_{2}\left(\frac{1}{2} d_{\min }\right)$ is given by

$$
P_{2}\left(\frac{1}{2} d_{\min }\right)=P_{2}\left(\frac{N_{\mathrm{cps}}}{4}\right)=p\left(\frac{N_{\mathrm{cps}}}{4}\right) \cdot \sum_{k=0}^{\left(\frac{N_{\mathrm{cps}}}{4}\right)^{-1}}\left(\left(\frac{N_{\mathrm{cps}}}{4}\right)_{k}-1+k\right) \cdot(1-p)^{k}
$$

where

$p=Q\left(\sqrt{\mathrm{SNR}_{\mathrm{c}}}\right)=Q\left(\sqrt{\frac{2 \cdot \mathrm{SNR}_{\mathrm{b}} \cdot N_{\mathrm{os}}}{N_{\text {cps_base }}}}\right)=Q\left(\sqrt{\frac{2 \cdot \mathrm{SNR}_{\mathrm{s}} \cdot N_{\mathrm{os}}}{N_{\text {cps_base }}} \cdot \frac{B_{\mathrm{s}}}{R}}\right)$, as the coherent OOK demodulation with matched filter through AWGN channel, $B_{\mathrm{s}}$ is the noise bandwidth, $R$ is the wake-up radio's data rate, and $\mathrm{SNR}_{\mathrm{s}}$ is the Signal-toNoise Ratio (SNR). Thus, the upper bound probability of a symbol (code word) error is given by

$$
P_{\mathrm{es}} \leqslant p\left(\frac{N_{\mathrm{cps}}}{4}\right) \cdot \sum_{k=0}^{\left(\frac{N_{\mathrm{cps}}}{4}\right)^{-1}}\left(\left(\frac{N_{\mathrm{cps}}}{4}\right)_{k}-1+k\right) \cdot(1-p)^{k}
$$

where $p=Q\left(\sqrt{\frac{2 \cdot \mathrm{SNR}_{\mathrm{s}} \cdot N_{\mathrm{os}}}{N_{\text {cps_base }}} \cdot \frac{B_{\mathrm{s}}}{R}}\right)$.

\section{Performance evaluation}

We first conduct empirical experiment to validate our prototype. Then, in terms of system performance of our design, we build a system model into a Matlab simulator to expose the system factors that affect the system performance. Furthermore, for exploring the energy and latency tradeoffs of multiple wake-up radios, we create an energy and latency model into a Matlab simulator to consider the total energy consumption and latency for a given number of nodes, including transmitter, receiver, and extra energy and latency including neighbor nodes overhearing, false wake-up, and retransmission. The final part of this section shows the comparison of our design with other wake-up radios.

\section{Empirical evaluation}

The empirical experiment to evaluate our prototype is designed as follows. The sender node sends symbol A and symbol B continuously using continuous wave (CW) mode at carrier frequency $916.3+0.1 \mathrm{MHz}$ and an antenna switch. The receiver node receives symbol $A$ and symbol B and computes the SER, in 1000 symbols for each round with symbol length $2.7 \mathrm{~ms}$, for different input signal power. The performance comparison for our wake-up radio with previously published wake-up radios and the IEEE 802.15.4 data radio is depicted in Table 1 . We have applied the same spreading code scheme into other wake-up radios to demonstrate fairly the comparison of our wake-up radio with other wake-up radios in Table 1 , Figure 5(a) and 5(b) regarding the SER, PER, power consumption, and latency. We determine the SER based on the bit error rate (BER) for multiple wakeup radios. The SER is given by $1-(1-\mathrm{BER})^{N_{\text {cps }} \text {, }}$ where $N_{\text {cps }}$ is the number of chips per symbol. The wake-up radio's PER at SNR PER $\left.{ }_{\text {wur }}\right|_{S N R}$ is given by

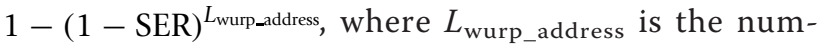
ber of symbols of a wake-up packet's address. Our design has a better wake-up packet error rate (PERwur $\left.\left.\right|_{\text {SNR }}\right)$ of 0.0159 at SNR $4 \mathrm{~dB}$ and a better sensitivity of $-122 \mathrm{dBm}$ at SNR $-4 \mathrm{~dB}$ with an assumed receiver noise floor of $-118 \mathrm{dBm}$.

\section{Simulations}

From Table 1, we find the empirical experimental results for our wake-up radio. In order to explore the performance of our spreading code scheme, we create a system model into a Matlab simulator. The system model uses our detection algorithm, in Additional file 1, Algorithm 1, to find out the potential factors that optimize the system performance. The simulation block diagram is shown in Figure 6. 
Table 1 Comparison of performance for our wake-up radio with other wake-up radios and data radio [12,14,15]

\begin{tabular}{|c|c|c|c|c|c|}
\hline \multirow{2}{*}{$\frac{\text { Related work }}{\text { Sensitivity }(\mathrm{dBm})}$} & \multicolumn{2}{|c|}{ This work } & \multirow{2}{*}{$\begin{array}{l}{[15]} \\
-110\end{array}$} & \multirow{2}{*}{$\begin{array}{l}{[12]} \\
-72\end{array}$} & \multirow{2}{*}{$\begin{array}{l}{[14]} \\
-84\end{array}$} \\
\hline & -122 & -114 & & & \\
\hline SER (\%) & 1 & 0.1 & 0.1 & 1.59 & 1.59 \\
\hline Data rate $(\mathrm{kbit} / \mathrm{s})$ & 0.37 & 0.37 & 20 & 100 & 100 \\
\hline Frequency $(\mathrm{GHz})$ & 0.916 & 0.916 & 0.9 & 2 & 2.4 \\
\hline$\left.P E R_{\text {wur }}\right|_{S N R}$ & $0.1485(-4 \mathrm{~dB})$ & $0.0159(4 \mathrm{~dB})$ & $0.016(8 \mathrm{~dB})$ & $0.226(46 \mathrm{~dB})$ & $0.226(34 \mathrm{~dB})$ \\
\hline
\end{tabular}

This paper achieves the highest sensitivity feature with the lowest packet error rate at a certain SNR
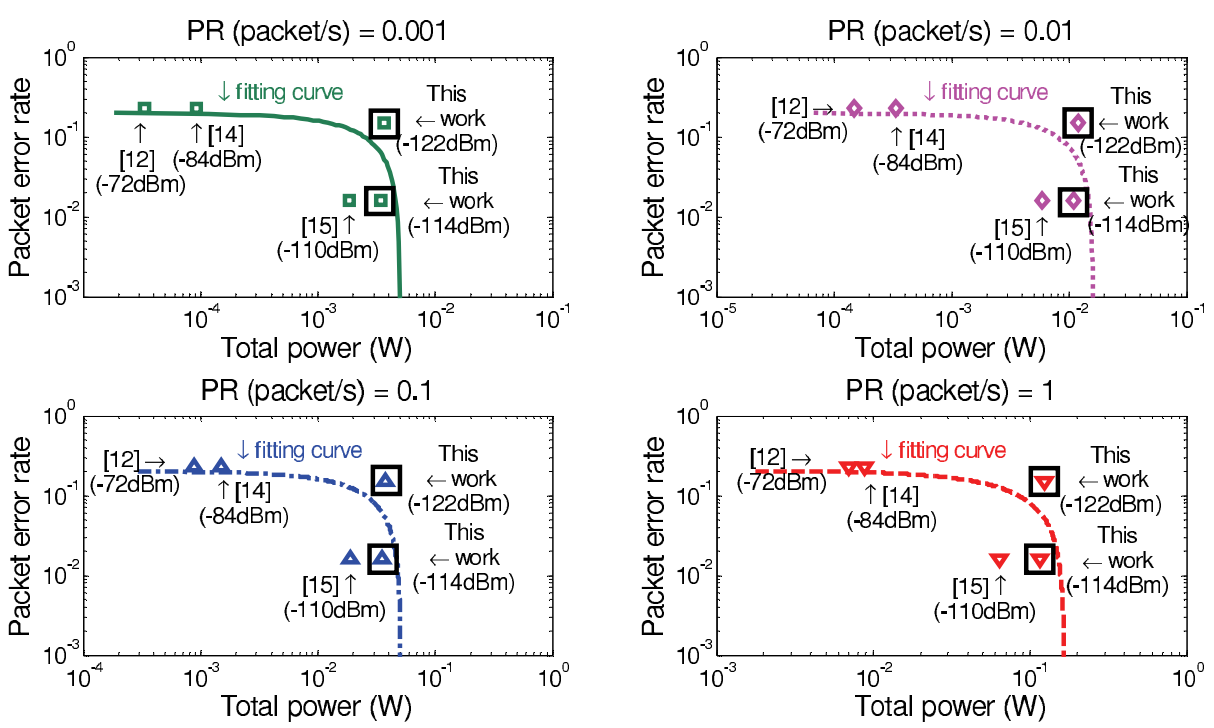

(a)
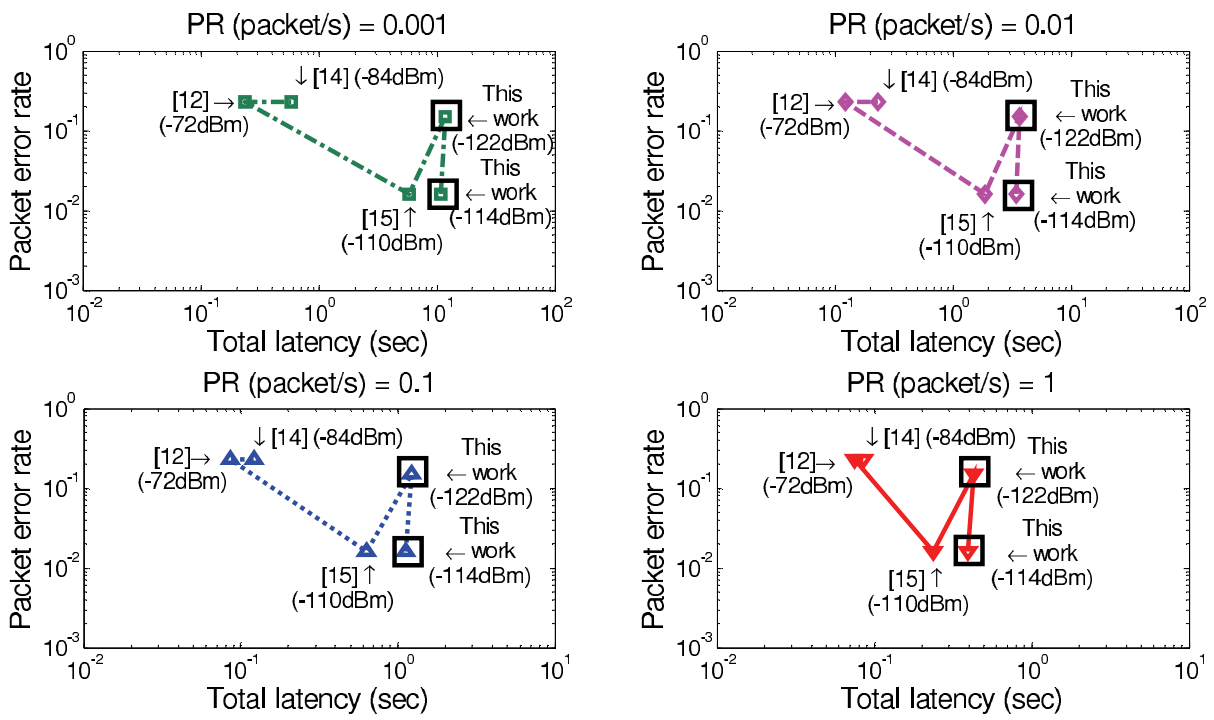

(b)

Figure 5 The comparison of energy and latency for multiple wake-up radios [12,14,15]. (a) Packet error rate versus total power. (b) Packet error rate versus total latency. 


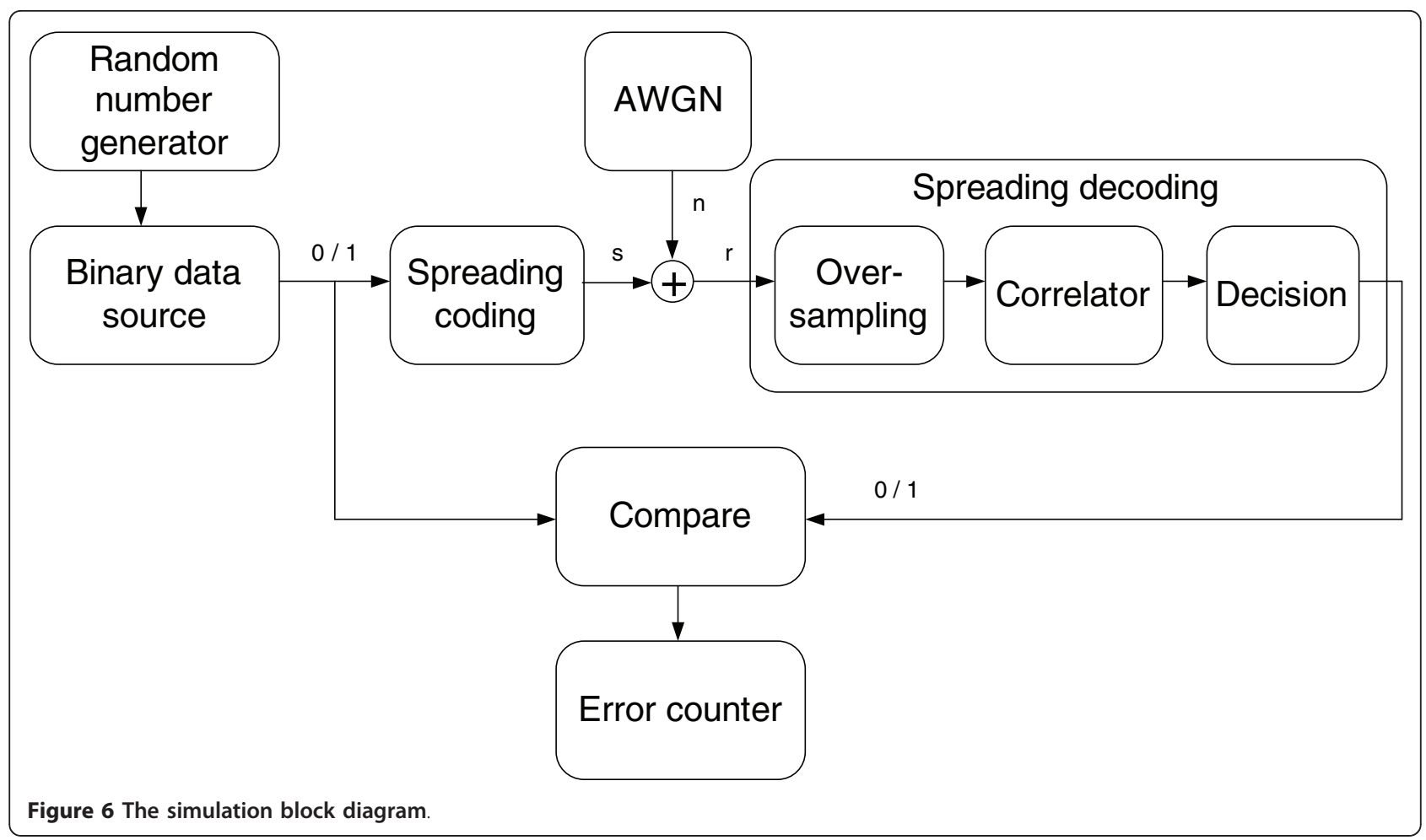

\section{Model}

Based on our measured results in the sensitivity versus SER experiment, we validate our theoretical analysis and system model, and draw the similar waterfall curves as measured results in Figure 7. In Figure 7, the SS stands for Spreading Spectrum, which represents our spreading code scheme. The experiment with 1000 symbols allows us to estimate the measured SER of $10^{-2}$ reliably. The difference between the simulated and empirical fitting curves might be the interference from other test equipments or the leakage signals from the transmitter in our Lab. The analytical curve shows the upper bound SER and is the ceiling of the simulated curve. Table 2 summarizes the theoretical analysis and simulation parameters for the system model of our wake-up radio.

From Figure 7, we can observe that when the SER is $10^{-3}$, the measured spreading code's SNR of $4 \mathrm{~dB}$ is better than the OOK's SNR of $10 \mathrm{~dB}$. Given a SER of $10^{-2}$, the measured spreading code's SNR is $-4 \mathrm{~dB}$ which is even much better than the OOK's SNR of $7 \mathrm{~dB}$ as our algorithm provides error-resilient capability to suppress the out-of-band and the in-band interference when the receiving signal strength is under the noise floor.

\section{Characterization}

We use the theoretical analysis and system model with different chips per symbol (CPS) to find out the influence of chips per symbol on system performance. The theoretical analysis and the simulation results are depicted in Figure 8 that shows when the CPS increases twice, the performance of the CPS increases up to $3 \mathrm{~dB}$ at a given SER of $10^{-4}$. We observe that optimal number of chips per symbol is 128 chips/symbol, for lower receiving signal power, providing the best sensitivity performance SNR $-18 \mathrm{~dB}$ at SER $1.13 \times 10^{-3}$. For this number of the CPS, we can detect the spreading code under the assumed noise floor $-118 \mathrm{dBm}$ with the cost of 1.01 $\mathrm{s}$ for total latency and $314 \mathrm{~mW}$ for total power dissipation at a wake-up packet rate (PR) of 1 packet/s.

Next, we explore the effect of the oversampling (OS) rate on system performance. We simulate the system model by varying OS rates in our simulator. The theoretical analysis and simulation results, shown in Figure 9, confirm that OS each chip suppresses the interference. We observe that when OS rate increases twice, the performance of the OS rate increases up to $3 \mathrm{~dB}$ at a given SER of $10^{-4}$. The optimal OS rate of 32 achieves the best sensitivity feature SNR $-18 \mathrm{~dB}$ at SER $1.13 \times 10^{-3}$. For this number of the OS rate, we can detect the spreading code under the assumed noise floor -118 $\mathrm{dBm}$. This causes $395 \mathrm{~ms}$ for total latency and 115.5 $\mathrm{mW}$ for total power dissipation at a wake-up PR of 1 packet/s.

\section{Tradeoffs}

We now evaluate multiple wake-up radios $[12,14,15]$ using our energy and latency model and our wake-up protocol with low power listening [3] to show the 


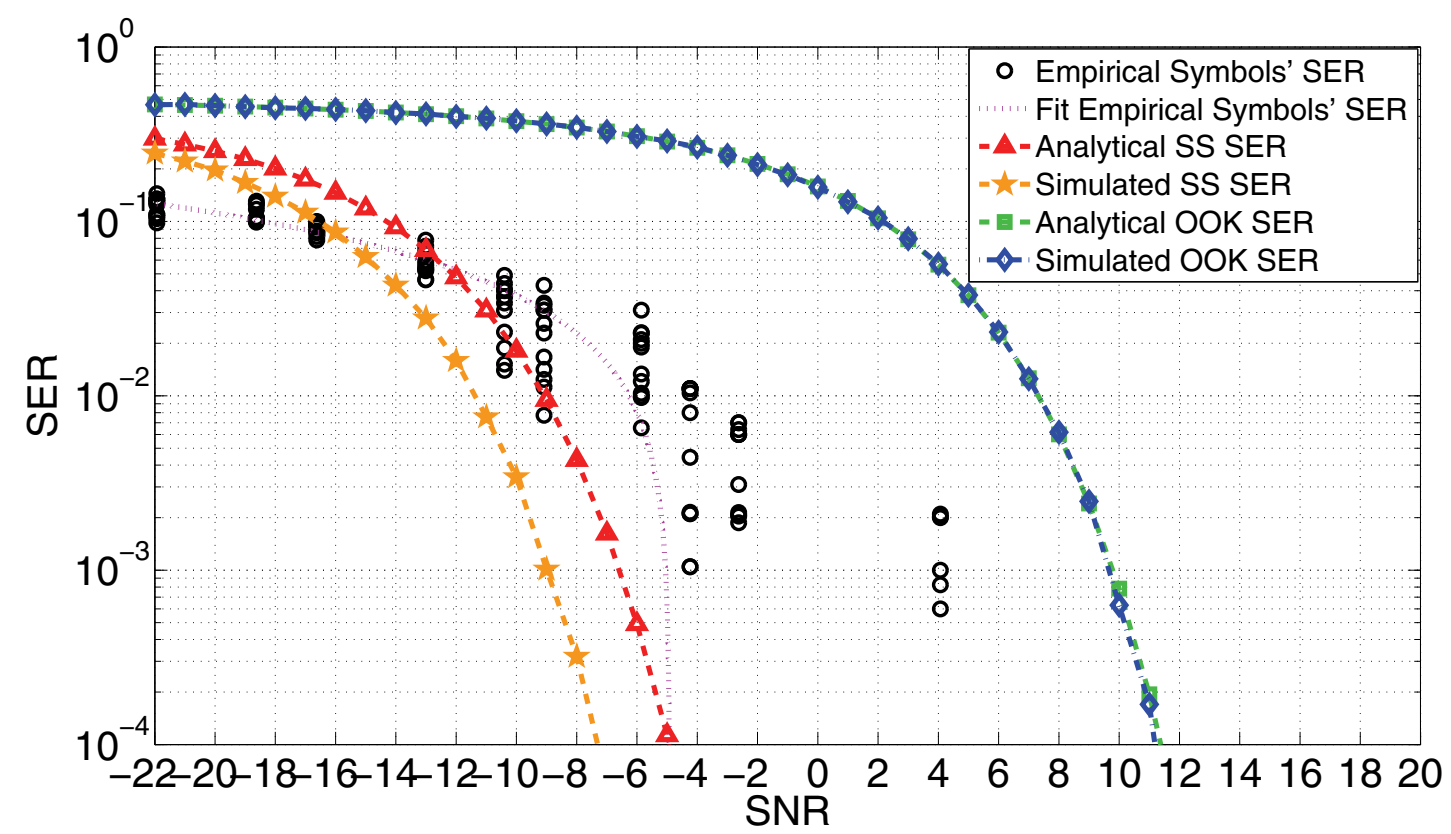

Figure 7 The SER versus SNR.

energy and latency tradeoffs at different PRs and for a given number of neighbor nodes $N$. Our energy and latency model enhances existing models [6], is generalizable to any wake-up radios, and serves as the basis for evaluating the power consumption and latency of multiple wake-up radios with different PERs for a given PR, a given number of neighbor nodes, and a given wake-up protocol, in order to determine the optimal PR setting. Our wake-up protocol is illustrated in Figure 10. Figure 10(a) shows our wake-up protocol using DC to listen the wake-up packets without false wake-up packet and false message. Figure 10(b) shows the retransmission from sender, when the false wake-up packet and false message occur. We observe that there are three possible cases based on false wake-up packets, false messages, and a successful wake-up. We discuss details later. We use differentiation operation [6] to find the optimal preamble time period for our wake-up protocol to achieve the lowest power dissipation for multiple wake-up

Table 2 The analysis and simulation parameters for our wake-up radio.

\begin{tabular}{lcc}
\hline Parameter & Value & Units \\
\hline Number of transmission bit $(N)$ & $10^{5}$ & bits \\
Number of chip per symbol $\left(N_{\text {cps }}\right)$ & 16 & chips/symbol \\
Number of oversampling $\left(N_{\text {os }}\right)$ & 4 & samples/chip \\
Noise bandwidth $\left(B_{\mathrm{s}}\right)$ & 6850 & $\mathrm{~Hz}$ \\
Wake-up radio's data rate $(R)$ & 370 & $\mathrm{bit} / \mathrm{s}$ \\
Scale value $\left(N_{\text {cps_base }}\right)$ & 16 & chips/symbol \\
\hline
\end{tabular}

radios. Table 3 summarizes the simulation parameters for energy and latency model of multiple wake-up radios $[12,14,15]$. We assume the number of wake-up radios $N_{\text {wur }}$ is half of the number of neighbor nodes $N$ as neighbor nodes are $N$ data radios including $\frac{N}{2}$. senders and $\frac{N}{2}$. receivers with the built-in wake-up radios.

We analyze the total power consumption in three cases to determine the total power consumption's expected value. The first case (case 1 with orange box) addresses the false wake-up packets. This case has two possible cases, case $1 \mathrm{a}$ and case $1 \mathrm{~b}$. One is that the error wake-up packet has been received by wake-up radios with matched wake-up ID addresses, the other one is that the error wake-up packet has been received by wake-up radios without any matched wake-up ID addresses. In the earlier case, the wake-up radios will acknowledge the sender as they receive the matched wake-up ID addresses. The sender will send the message to wake-up radios. The wake-up radios find that the message's ID address does not match their own wakeup ID addresses. Then, the wake-up radios send retransmission requests to sender, and then they go to sleep. Regarding the message's ID address, it is the same as wake-up ID address. The wake-up radio receives the wake-up signal's wake-up ID address and the message's ID address from sender. It can compare the wake-up ID address with the message's ID address to know if both of them are correct or one of them is incorrect. If the 


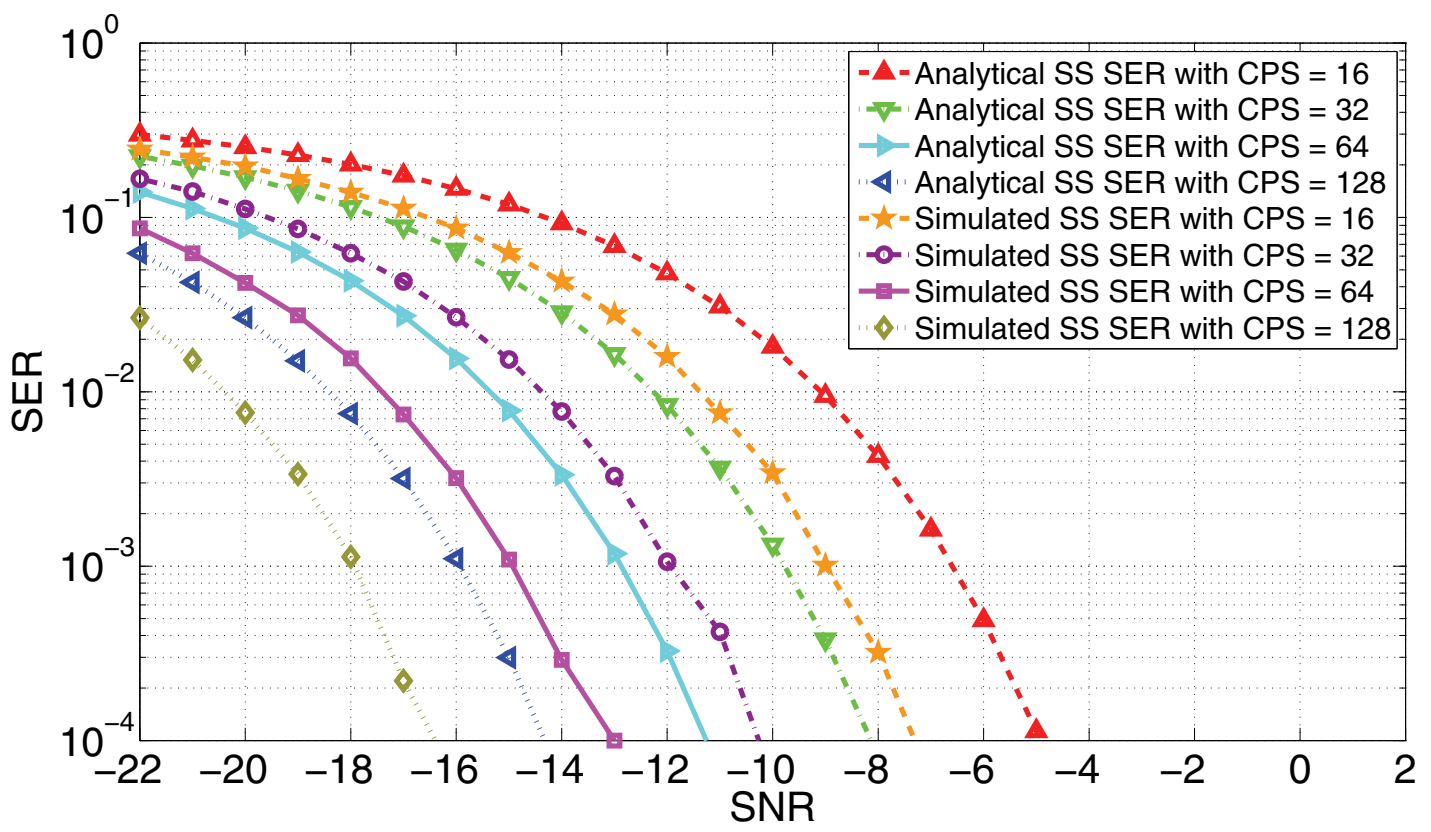

Figure 8 The SER versus SNR with different CPS

wake-up ID address matches the message's ID address, then both of them are correct. If wake-up ID address does not match the message's ID address, then one of them is incorrect. In this case, the wake-up radio will request retransmission. In the latter case, the wake-up radios will not acknowledge the sender, as mismatched wake-up ID addresses, and go to sleep. As the wake-up receiver is separate from the main data receiver, they have different bit error rates. In case 2 , we discuss the main data receiver's error from the noise. If the wake-up ID is correct and

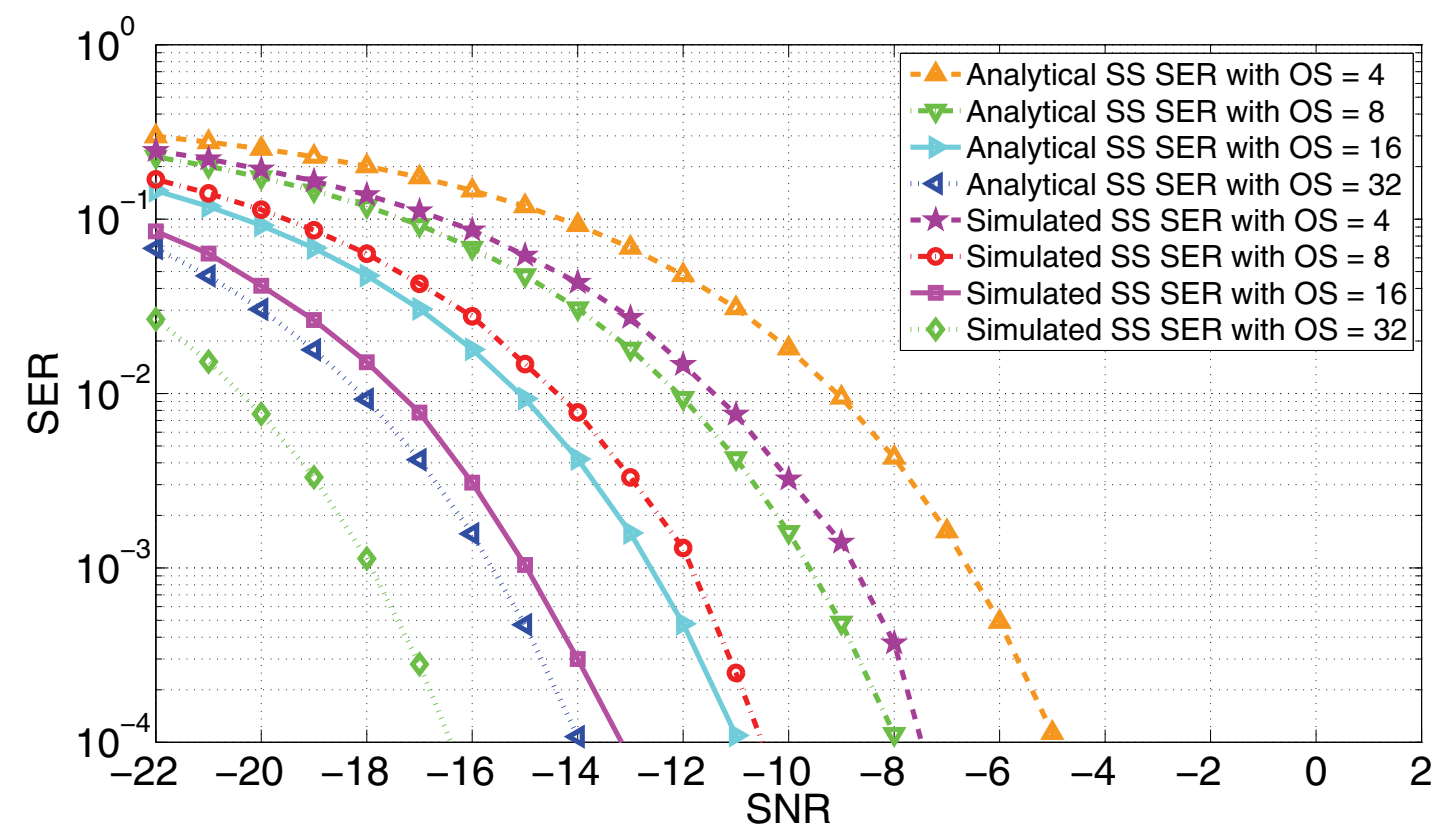

Figure 9 The SER versus SNR with different OS rates. 


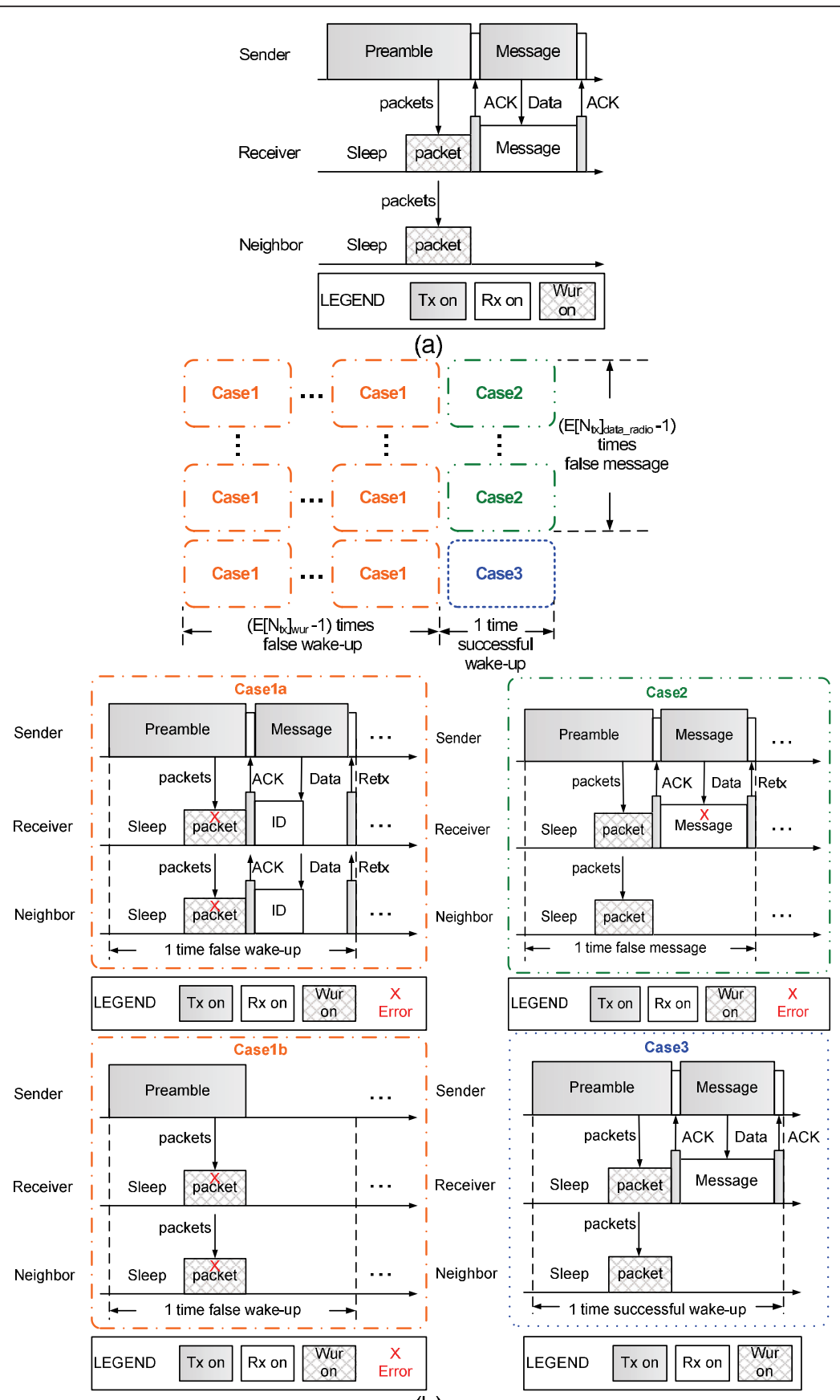

(b)

Figure 10 The wake-up protocol with DC. (a) Without false wake-up packet and false message. (b) With false wake-up packet and false message.

the correct wake-up receiver ACKs, then the message received by the main data receiver might be incorrect as the main data receiver has its own bit error rate, which is different with the wake-up receiver. The second case (case 2 with green boxes) addresses the false message. The correct wake-up radio receives the false message and sends retransmission request to sender. Other wake-up radios go to sleep after they receive the correct wake-up packets. The third case (case 3 with blue boxes) addresses a successful wake-up packet and a successful message. Only the correct wake-up radio will acknowledge the sender as it receives a correct wake-up packet and a correct message. Other wake-up radios go to sleep after they receive the correct wake-up packets. 
Table 3 The simulation parameters for multiple wake-up radios $[12,14,15]$

\begin{tabular}{|c|c|c|c|}
\hline $\begin{array}{l}\text { Wake-up } \\
\text { radio }\end{array}$ & Parameter & Value & Units \\
\hline \multirow[t]{10}{*}{ All } & Number of neighbors $(N)$ & 10 & nodes \\
\hline & Number of wake-up radios ( $\left.N_{\text {wur }}\right)$ & 5 & nodes \\
\hline & Wake-up packet length $\left(L_{\text {wurp }}\right)$ & 40 & symbols \\
\hline & Wake-up address length ( $L_{\text {wurp_address }}$ ) & 16 & symbols \\
\hline & Wake-up information length $\left(L_{\text {wurinf }}\right)$ & 24 & symbols \\
\hline & Data radio supply voltage $\left(V_{c c d}\right)$ & 3.3 & V \\
\hline & Data radio transmit current $\left(I_{\mathrm{td}}\right)$ & 25 & $\mathrm{~mA}$ \\
\hline & Data radio receive current $\left(I_{\mathrm{rd}}\right)$ & 9.2 & $\mathrm{~mA}$ \\
\hline & $\begin{array}{l}\text { Data radio sending message time } \\
\left(T_{\text {msg }}\right)\end{array}$ & 50.8 & $\mathrm{~ms}$ \\
\hline & Data radio sending node ID time $\left(T_{\mathrm{id}}\right)$ & 1.18 & ms \\
\hline \multirow[t]{6}{*}{ This work } & Supply voltage $\left(V_{\text {ccwur }}\right)$ & 3.3 & V \\
\hline & Receive current (I wur $)$ & 9.5 & $\mathrm{~mA}$ \\
\hline & Data rate $\left(R_{\text {wur }}\right)$ & 370 & $\mathrm{bit} / \mathrm{s}$ \\
\hline & $\begin{array}{l}\text { Wake-up time from shut down } \\
\left(T_{\text {stwur }}\right)\end{array}$ & 1.1 & ms \\
\hline & $\begin{array}{l}\text { Symbol error rate at }-114 \mathrm{dBm} \\
\left(\mathrm{SER}_{\text {wurl }}\right)\end{array}$ & 0.1 & $\%$ \\
\hline & $\begin{array}{l}\text { Symbol error rate at }-122 \mathrm{dBm} \\
\text { (SER }\end{array}$ & 1 & $\%$ \\
\hline \multirow[t]{3}{*}{ [15] } & Data rate $\left(R_{\mathrm{d}}\right)$ & 20 & $\mathrm{kbit} / \mathrm{s}$ \\
\hline & Wake-up time from shut down $\left(T_{\text {std }}\right)$ & 380 & $\mu s$ \\
\hline & Symbol error rate $\left(S E R_{d}\right)$ & 0.1 & $\%$ \\
\hline \multirow[t]{4}{*}{ [12] } & Receive power $\left(P_{\text {ruif }}\right)$ & 52 & $\mu \mathrm{W}$ \\
\hline & Data rate $\left(R_{\text {uif }}\right)$ & 100 & $\mathrm{kbit} / \mathrm{s}$ \\
\hline & Wake-up time from shut down ( $\left.T_{\text {stuif }}\right)$ & 0 & $\mu s$ \\
\hline & Bit error rate (BER uif $)$ & 0.1 & $\%$ \\
\hline \multirow[t]{4}{*}{ [14] } & Receive power $\left(P_{\text {rulp }}\right)$ & 500 & $\mu \mathrm{W}$ \\
\hline & Data rate $\left(R_{\text {ulp }}\right)$ & 100 & $\mathrm{kbit} / \mathrm{s}$ \\
\hline & Wake-up time from shut down $\left(T_{\text {stulp }}\right)$ & 5.24 & $\mu s$ \\
\hline & Bit error rate $\left(B E R_{\text {ulp }}\right)$ & 0.1 & $\%$ \\
\hline
\end{tabular}

The total power consumption $P_{\text {total }}$ is given by

$$
\begin{aligned}
P_{\text {total }}= & \left(\left(q_{\text {case1a }} \cdot P_{\text {case } 1 \mathrm{a}}+q_{\text {case1b }} \cdot P_{\text {case } 1 \mathrm{~b}}\right)+\right. \\
& \left.q_{\text {case2 }} \cdot P_{\text {case2 }}+q_{\text {case3 }} \cdot P_{\text {case3 } 3}\right) \cdot E\left[N_{\text {tx }}\right]_{\text {case3 }}+P_{\text {idle }}
\end{aligned}
$$

where $q_{\text {case la }}$ is the probability of case $1 \mathrm{a}, q_{\text {case } 1 \mathrm{~b}}$ is the probability of case $1 \mathrm{~b}, q_{\text {case } 2}$ is the probability of case $2, q_{\text {case } 3}$ is the probability of case $3, P_{\text {case } 1 \mathrm{a}}$ is the power consumption of case $1 \mathrm{a}, P_{\text {case }} 1 \mathrm{~b}$ is the power consumption of case $1 \mathrm{~b}, P_{\text {case } 2}$ is the power consumption of case $2, P_{\text {case } 3}$ is the power consumption of case $3, P_{\text {idle }}$ is the average power consumption when there is not any transmission and all wake-up radios look for a wake-up packet within DC, and $E\left[N_{\text {tx }}\right]_{\text {case } 3}$ is the expected transmission times in case 3 , which means that a wake-up packet and a message both are successful, given by

$$
\begin{aligned}
E\left[N_{\mathrm{tx}}\right]_{\text {case3 }} & =\frac{1}{q_{\text {case3 }}}=\frac{1}{\left(1-\mathrm{PER}_{\mathrm{wur}}\right) \cdot\left(1-\mathrm{PER}_{\mathrm{data} \_ \text {radio }}\right)} \\
& =E\left[N_{\mathrm{tx}}\right]_{\mathrm{wur}} \cdot E\left[N_{\mathrm{tx}}\right]_{\text {data_radio }}
\end{aligned}
$$

where $E\left[N_{\mathrm{tx}}\right]_{\text {wur }}$ is the expected transmission times for the wake-up radio's wake-up packet, given by $\frac{1}{\left(1-\mathrm{PER}_{\mathrm{wur}}\right)}[29]$, and $E\left[N_{\mathrm{tx}}\right]_{\text {data_radio }}$ is the expected transmission times for the data radio's message, given by $\frac{1}{\left(1-\text { PER }_{\text {data_radio }}\right)}$, where $P E R_{\text {wur }}$ is the wake-up radio's PER, PER data_radio is the data radio's PER.

The optimal preamble time duration $T_{\text {preamble_optimal }}$ is given by

$$
\begin{aligned}
& \text { Let } \frac{\partial}{\partial T_{\text {preamble }}} P_{\text {total }}=0 \text { to find the optimal } T_{\text {preamble }}, \\
& T_{\text {preamble_optimal }} \\
& T_{\text {preamble_optimal }}=\sqrt{\frac{P_{\text {wur }} \cdot T_{\text {wurpacket }} \cdot N_{\text {wur }}}{\left(\left(V_{\mathrm{ccd}} \cdot I_{\mathrm{td}}\right)+\frac{N_{\text {wur }} \cdot P_{\text {wur }}}{2}\right) \cdot P R \cdot E\left[N_{\text {tx }}\right]_{\text {case } 3}}}
\end{aligned}
$$

where $T_{\text {preamble }}$ is the preamble time duration, when a sender sends the consecutive wake-up packets to a receiver's wake-up radio, $P_{\text {wur }}$ is the wake-up radio's power consumption, $\left(V_{\mathrm{ccd}} \cdot I_{\mathrm{td}}\right)$ is the data radio's transmission power, and $T_{\text {wurpacket }}$ is the wake-up packet's time duration.

The simulation results of the energy tradeoffs at different PRs for a given number of neighbor nodes $N$ are shown in Figure 5(a). The relationships between total power consumption and system reliability are like the fitting waterfall curves. The fitting waterfall curves mean a lower reliability system has a lower power consumption, while a higher reliability system has a similar power consumption as other lower reliability systems at high PR of 1 packet/s. The fitting waterfall curves predict that the future wake-up radios' power and reliability points will be close to the fitting waterfall curves at varying PR. We observe that the wake-up radios $[12,14]$ have similar power consumptions at the high PR 0.1 and 1 packet/s as they both have high PERs. The reason is that previous wake-up radios provide a low power consumption at the cost of short radio range, high deployment density, low system reliability, high PER, and high extra energy, from false wake-up and retransmission, when PR increases. In contrast, our wake-up radio provides most reliable performance, reduces deployment density, and has the total power consumption of $115.3 \mathrm{~mW}$ that is approximating to other low power wake-up radios' power consumption at high PR of 1 packet/s. Although our design's power consumption is larger than other wake- 
up radios, it reduces deployment density, and is still acceptable and more suitable to sensor networks within a long radio range.

In terms of the relationship between total latency and system reliability, the simulation results are shown in Figure 5(b). As all the wake-up radios use the same wake-up protocol, we apply the differentiation operation [6] to find the optimal preamble time period for our wake-up protocol, in order to achieve the lowest power dissipation for multiple wake-up radios. The total latency $T_{\text {total }}$ is given by

$$
T_{\text {total }}=E\left[N_{\mathrm{tx}}\right]_{\text {case } 3} \cdot\left(T_{\text {preamble_optimal }}+T_{\mathrm{msg}}\right)
$$

where $T_{\mathrm{msg}}$ is the message time duration when a transmitter sends a message to a receiver's data radio. Therefore, when the PR changes, each PR has its optimal preamble time period and extra latency, caused by its false wake-up and retransmission, larger at higher PR. We observe that the wake-up radios $[12,14]$ have similar latency at the high PR 0.1 and 1 packet/s as they both have high PERs. Our wake-up radio has a total latency of $394.5 \mathrm{~ms}$, at a PR of 1 packet/s, which is close to other low sensitivity wake-up radios' total latency. It is also suitable when the PR increases. Although our design has a larger latency than other low power wake-up radios, it increases the radio range by up to 4 times, reduces deployment density, and is more suitable to sensor networks with less deployment density within a long radio range than other short range wakeup radios.

\section{Performance comparison}

The performance of our design is compared with previously published wake-up radios and data radio [11-13,15] in Figure 11. Other wake-up radios provide a short radio range and increase deployment density that is not suitable to the sensor networks applications. However, our design provides high sensitivity feature, which provides a long radio range and reduces deployment density. Our design uses the spreading code scheme to achieve the $13 \mathrm{~dB}$ of coding gain at SER $1 \%$, while it has better sensitivity, up to $4 \mathrm{~dB}$ at a given SER of $0.1 \%$, than the data radio. The tradeoff is that our design has the larger power consumption and latency than other wake-up radios, when the PR decreases.

\section{Discussions}

As previous related wake-up radios provide low sensitivity feature result in higher deployment density of sensor network, the sensitivity of wake-up radios should be improved to reduce the deployment density of sensor networks. Therefore, our design is more suitable to sensor networks than other wake-up radios.

Using an active component and a spreading code correlation algorithm, our high sensitivity wake-up radio provides good rejection of out-of-band and inband interference and reduces the false alarm rate to

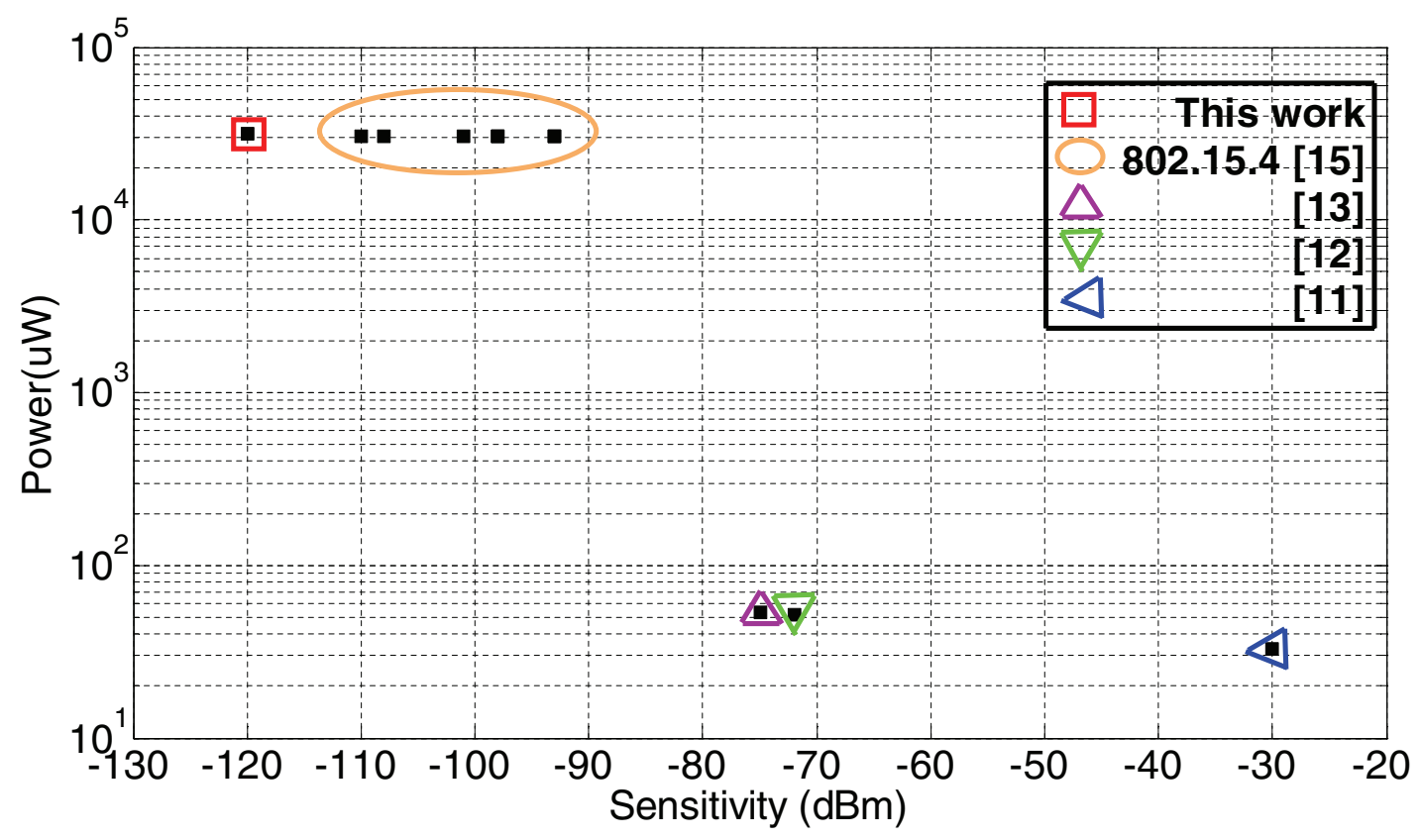

Figure 11 The performance of our wake-up radio is compared with previously published wake-up radios and data radio [11-13,15]. 
reduce power consumption and latency. As for other wake-up radios that use passive components, such as diode rectifiers, they detect out-of-band signals, increasing their false wake-up packets, power consumption and latency.

From the performance evaluation described above, we observe that when our wake-up radio, with a spreading code set $\mathrm{SC} 1$, uses the configuration of the OS rate of 32 and the PR of 1 packet/s, our design has better SER of $1.13 \times 10^{-3}$ in a given SNR of $-18 \mathrm{~dB}$, which means the high sensitivity $-136 \mathrm{dBm}$. It also can achieve more approximate power dissipation and a similar latency as other wake-up radios, provides up to 16 times radio range, reduces deployment density, and is more suitable and reliable to sensor networks than other wake-up radios.

Choosing the empirical configuration, of the CPS of 16 chips/symbol, the OS rate of 4 and the DC of $1 \%$ for sampling 3 symbols per $\mathrm{DC}$, achieves the best empirical sensitivity feature SNR $-4 \mathrm{~dB}$ at SER $10^{-2}$, which means the sensitivity $-122 \mathrm{dBm}$, while it performs a long radio range about $1 \mathrm{~km}$, in free space path loss conditions [30], when the sender's transmission power is $0 \mathrm{dBm}$.

In terms of the benefits from our article for other wake-up radios, our empirical configuration provides up to $13 \mathrm{~dB}$ of empirical coding gain at SER $10^{-2}$. Previous wake-up radios can apply our spreading code scheme to improve their SER, PER, system reliability, up to $13 \mathrm{~dB}$ of sensitivity, and up to 4 times communication range. This can provide them with lower extra power dissipation and latency from fewer false wake-ups and retransmissions.

The performance analysis results also empower the wake-up radio designers to consider various choices for the expected long communication range and SER requirement of the desired applications, based on the trade offs with power and latency.

\section{Additional material}

Additional file 1: Algorithm 1. Spreading code detection.

\begin{abstract}
Abbreviations
AWGN: Additive White Gaussian Noise; BER: bit error rate; CPS: chips per symbol; CW: continuous wave; DC: duty cycle; FEC: forward error correction; LPF: low pass filter; MCU: micro-controller unit; OS: oversampling; OOK: OnOff Keying; PR: packet rate; PER: packet error rate; RFID: Radio-frequency identification; SNR: Signal-to-Noise Ratio; SER: symbol error rate.
\end{abstract}

\section{Acknowledgements}

The authors thank Christian Richter, Brano Kusy, Leslie Overs, Mikhail Afanasyev, Damien O'Rourke, Tim Wark, Wen Hu, Darren Moore, Morten Hansen, Kevin Klues, Paul Flick, and Brett Wood in ICT Centre, Commonwealth Scientific and Industrial Research Organization (CSIRO), Australia for fruitful discussions.

\section{Author details}

'Department of Electrical Engineering, National Taiwan University of Science and Technology, 2F, EE, No. 43, Sec. 4, Keelung Rd, Taipei 106, Taiwan ${ }^{2}$ Autonomous Systems Laboratory, ICT Centre, CSIRO, 1 Technology Court, Pullenvale, Qld 4069, Australia ${ }^{3}$ Wireless Laboratory, ICT Centre, CSIRO, Corner of Pembroke Street \& Vimiera Road, Marsfield, NSW, 2122, Australia

\section{Competing interests}

The authors declare that they have no competing interests.

Received: 2 April 2011 Accepted: 30 June 2011 Published: 30 June 2011

\section{References}

1. R Jurdak, AG Ruzzelli, GMP O'Hare, Multi-hop RFID wake-up radio: design, evaluation and energy tradeoffs. in Proceedings of the 17th International Conference on Computer Communications AND Networks (ICCCN), August 2008

2. R Jurdak, AG Ruzzelli, GMP O'Hare, Radio sleep mode optimization in wireless sensor networks. IEEE Trans Mob Comput. 9(7), 1-17 (2010)

3. J Polastre, J Hill, D Culler, Versatile low power media access for wireless sensor networks. in SenSys '04: Proceedings of the 2nd international conference on Embedded networked sensor systems, ACM, New York, NY, USA, 95-107 (2004)

4. CC Enz, A El-Hoiydi, JD Decotignie, V Peiris, WiseNET: an ultralow-power wireless sensor network solution. Computer. 37, 62-70 (2004)

5. W Ye, F Silva, J Heidemann, Ultra-low duty cycle MAC with scheduled channel polling. in SenSys '06: Proceedings of the 4th international conference on Embedded networked sensor systems, ACM, New York, NY, USA, 321-334 (2006)

6. BVd Doorn, W Kavelaars, K Langendoen, A prototype low-cost wakeup radio for the $868 \mathrm{MHz}$ band. Int J Sensor Netw. 5(1), 22-32 (2009)

7. W Che, W Chen, D Meng, X Wang, X Tan, N Yan, H Min, Power management unit for battery assisted passive RFID tag. Electron Lett. 46(8), 589-590 (2010). doi:10.1049/el.2010.0113

8. J Ansari, D Pankin, P Mähönen, Radio-triggered wake-ups with addressing capabilities for extremely low power sensor network applications. in PIMRC, $1-5$ (2008)

9. J Ansari, D Pankin, P Mähönen, Radio-triggered wake-ups with addressing capabilities for extremely low power sensor network applications. Int J Wireless Inform Netw. 16(3), 118-130 (2009). doi:10.1007/s10776-009-0100-6

10. $\mathrm{P}$ Le-Huy, S Roy, Low-power wake-up radio for wireless sensor networks. Mob Netw Appl. 15(2), 226-236 (2010). doi:10.1007/s11036-009-0184-3

11. P Koskela, M Valta, Simple wake-up radio prototype, in ACM HotEMNETS 2010 Workshop, 2010. HotEMNETS 2010. Embedded Networked Sensors, 28-29," June 2010, pp. 1-5

12. NM Pletcher, S Gambini, J Rabaey, A $52 \mu \mathrm{W}$ wake-up receiver with $-72 \mathrm{dBm}$ sensitivity using an uncertain-IF architecture. IEEE J Solid-State Circuits. 44(1), 269-280 (2009)

13. X Yu, J-S Lee, C Shu, S-G Lee, A $53 \mu \mathrm{W}$ super-regenerative receiver for 2.4 GHz wake-up application. in Microwave Conference, 2008. APMC 2008. Asia-Pacific, 16-20, Dec. 2008, pp. 1-4

14. S Drago, F Sebastiano, LJ Breems, DMW Leenaerts, KAA Makinwa, B Nauta, Impulse based scheme for crystal-less ULP radios. IEEE Trans Circuits Syst I: Regul Pap. 56(5), 1041-1052 (2009)

15. Atmel, AVR low power $700 / 800 / 900 \mathrm{MHz}$ Tranceiver for IEEE 802.15.4-2006, IEEE 802.15.4c-2009, Zigbee, 6LoWPAN, and ISM Applications AT86RF212, (2010). http://www.atmel.com/dyn/resources/prod_documents/doc8168.pdf. Accessed 25 Oct 2010

16. S Von der Mark, M Huber, G Boeck, Design concepts and first implementations for $24 \mathrm{GHz}$ wireless sensor nodes. J Commun. 2(6), 1-5 (2007)

17. I Demirkol, C Ersoy, E Onur, Wake-up receivers for wireless sensor networks: benefits and challenges. IEEE Wireless Commun. 16(4), 88-96 (2009)

18. H Kim, H Cho, Y Xi, M Kim, S Kwon, J Lim, Y Yang, CMOS passive wake-up circuit for sensor network applications. Microw Opt Technol Lett. 52(3), 597-600 (2010). doi:10.1002/mop.24982

19. Intelleflex (2010), The Intelleflex XC3 Technology ${ }^{\text {TM }}$ Platform. An Implementation of the New ISO Class C Standard. http://www.intelleflex. com/downloads/white-papers/Intelleflex-XC3-ISO-C3-White-Paper.pdf. Accessed 25 Oct 2010 
20. P Kolinko, LE Larson, Passive RF receiver design for wireless sensor networks, in Microwave Symposium, 2007. IEEE/MTT-S International, 567-570 (2007)

21. L Shan, $T$ Y Yunjian, Z Qin, Passive wake-up scheme for wireless sensor networks. in Second International Conference on Innovative Computing, Information and Control. ICICIC '07, 507-507 (2007)

22. DP Pereira, W Dias, M Braga, R Barreto, CMS Figueiredo, V Brilhante, Model to integration of RFID into wireless sensor network for tracking and monitoring animals, in 11 th IEEE International Conference on Computational Science and Engineering, CSE '08, 16-18, July 2008, pp. 125-131

23. CL Fok, GC Roman, C Lu, Mobile agent middleware for sensor networks: an application case study, in Fourth International Symposium on Information Processing in Sensor Networks, IPSN 2005, 15, April 2005, pp. 382-387

24. J Reid, JMG Nieto, T Tang, B Senadji, Detecting relay attacks with timingbased protocols, in Proceedings of the 2nd ACM symposium on Information, Computer and Communications Security, ACM, Singapore, 204-213 (2007). doi:10.1145/1229285.1229314

25. E Shih, P Bahl, MJ Sinclair, Wake on wireless: an event driven energy saving strategy for battery operated devices. in Paper presented at the Proceedings of the 8th annual international conference on Mobile computing and networking (Atlanta, Georgia, USA 2002)

26. CSIRO, Fleck3B. http://150.229.98.68/content/equipment/fleck3b (2010). Accessed 10 Dec 2010

27. Micrel (2008), MICRF221 3.3V, QwikRadio 850MHz to $950 \mathrm{MHz}$ Receiver. www.micrel.com/_PDF/micrf221.pdf. Accessed 25 Oct 2010

28. JG Proakis, Digital communications/John G. Proakis, McGraw-Hill Series in Electrical Engineering, Communications and Information Theory (McGrawHill New York 1983). Accessed from http://nla.gov.au/nla.cat-vn272371

29. A Woo, T Tong, D Culler, Taming the underlying challenges of reliable multihop routing in sensor networks, in SenSys '03: Proceedings of the 1st international conference on Embedded networked sensor systems, ACM, Los Angeles, California, USA, 14-27 (2003). doi:citeulike-articleid:3488470

30. J Zyren, A Petrick (1998), Tutorial on Basic Link Budget Analysis. Application Note AN9804, Harris Semiconductor, http://citeseerx.ist.psu.edu/viewdoc/ download?doi=10.1.1.165.4949\&rep=rep1\&type=pdf. Accessed 25 Oct 2010

doi:10.1186/1687-1499-2011-26

Cite this article as: Shih et al: High sensitivity wake-up radio using spreading codes: design, evaluation, and applications. EURASIP Journal on Wireless Communications and Networking 2011 2011:26.

\section{Submit your manuscript to a SpringerOpen ${ }^{\mathcal{O}}$ journal and benefit from:}

- Convenient online submission

- Rigorous peer review

- Immediate publication on acceptance

- Open access: articles freely available online

- High visibility within the field

- Retaining the copyright to your article

Submit your next manuscript at $\gg$ springeropen.com 